\title{
PROPOSAL FOR THE CLASSIFICATION OF CRITICAL POINTS BY ORDER
}

\author{
T.S. CHANG*, A. HANKEY and H.E. STANLEY \\ Physics Department, Massachusetts Institute of Technology, Cambridge, Massachusetts 02139, USA
}

Received 20 March 1973

\begin{abstract}
Critical points of complex thermodynamic systems are classified by their order 0 , defined here for the first time. A convenient notation is developed for such points.
\end{abstract}

The geometric approach to phase transitions in multicomponent systems [1], which was used by Griffiths to propose the existence of tricritical points [2], naturally leads one to pose the following question: "In more complex systems, with more than three field or field-like $\dagger^{1}$ variables, what sort of more complex critical points and critical phenomena can be expected?" In this work we propose the classification of critical points by their order, denoted by an index 0 , which we discuss below. For a particular space $\dagger^{2}$ of critical points (denoted CRS in ref. [1]) the value of $O$ depends only upon the topological properties of that CRS in an appropriately general space of field variables.

A tricritical point was defined [2] as a point where three lines of ordinary critical points meet. Thus, the dimensionality of a space of tricritical points is at least one less than the dimensionality of spaces of ordinary critical points which intersect there. Spaces of ordinary critical points are known to be of dimension $d$ two less than the total number of field variables $n$; hence a space of tricritical points is of dimension $d \leqslant$ $n-3$. If $n=4$ we may have lines of tricritical points, for $n=5$, surfaces, and so on.

A point of intersection of lines of tricritical points will be as different from a tricritical point as a tricritical point is from a simple critical point. This can be clearly seen by considering the topology at such a

$f^{1}$ The term field-like variables refers to quantities, such as parameters in a Hamiltonian, which do not suffer a discontinuity across a coexistence surface. Hereafter the term field-variable will mean both sorts of variable. See also Ief. [1].

$t^{2}$ In this context the word space refers to any line, surface, or higher dimensional entity of critical points. They are all smooth subspaces of the total space of field and field-like variables. point. At a line of tricritical points, three surfaces of critical points meet, whereas at the special point several (more than three) distinct surfaces of critical points converge on the special point, each surface being bounded by two of the lines of tricritical points.

Such a point, being qualitatively different, must be distinguished from ordinary critical points and from tricritical points. To accomplish this, we propose a definite number $O$, called the order, associated with each different kind of critical point and defined as follows. One can define ordinary critical points to be of order $\mathrm{O}=2$, and a special point where lines of order $\mathrm{O}$ intersect to be a critical point of order $\mathrm{O}+1$ $(0 \geqslant 2)$. Thus a tricritical point is of order $0=3$, while a point of intersection of lines of tricritical points is of order $\mathrm{O}=4$.

In general, the dimensionality of spaces of critical points can be larger than zero or one, but the dimensionality, $d$, of a space of critical points of order $O$ is always less than the dimensionality of the spaces of critical points of order $0-1$ which intersect at it. Griffiths and Wheeler [1] reasoned that the dimensionality of a space of ordinary critical points is $(n-2)$; the value of $d$ for arbitrary $O$ therefore satisfies

$d \leqslant n-0$

where $n$ is the total number of field (and field-like) variables available.

A specific example which demonstrates the impor-

Work supported by the NSF, ONR, AFOSR, NASA, and the MIT Laboratory for Nuclear Science. This work forms a portion of a Ph.D. thesis submitted to the Physics Department of MIT by A. Hankey.

*Permanent address: Riddick Laboratories, North Carolina State University, Raleigh, N.C., 27607, USA. 
tance of distinguishing the order of a critical pomt from the number of critical lines meeting there is the retracritical point $|3|$ of Nagle and Bonner. This is at point of order $O=3$ with field variables $\left(H, H_{\mathrm{x}}, \pi\right)$ where $H_{s 1}$ is the staggered magnetic field. When in field-like variable (the ratio of short-range to longrange interaction strengths) is also included. this be comes part of a line of tricritical points. $\mathrm{kq}$. (1) hecomes an equality since $n=4$ and $0=3$. The tetracru ical point is simply a point on a smooth line of tricit ical pointst ${ }^{3} \quad$ it is of order $0=3$ and nol $0=+$ This reasoning corroborates the fact. noted by Nagle and Bonner, that the exponents at the tetracritical point are the same as at the tricritical points.

To be concise we now introduce a notation fon critical points of arbitrary order. This notation is an extension of that of ref. [1]. As proposed there. the kt ters CRS denote a space of eritical points, but now the order $O$ will begiven by a preceding superseript, and the dimensionality $d$ by a subscript ( $O_{(\mathrm{RS}}$ ). Thus at tricritical point, which is a point (space of dimension zero) of order 3 is denoted by a ${ }^{3} \mathrm{CRS}_{(1)}$. A line of ordinary critical points is a ${ }^{2} \mathrm{CRS}_{1}$ : three ${ }^{2} \mathrm{CRS}_{1}$ need at a ${ }^{3} \mathrm{CRS}_{0}$.

Four field or field-like variables are needed to achieve a critical point of order 4 . Examples of systems exhibiting a critical point of order four are the following: (i) a three-dimensional lsing model with variable interaction $R J$ between planes; here the criti cal point of order four occurs in the limit $R \rightarrow(\mathrm{f}$ on the temperature axis [i.e. at the point $R=H=H_{\mathrm{s}}=$ 0 , and $T=T_{c}(R=0)$ ], (ii) a variation of the NagleBonner model with the long-range interaction acting

$\dot{7}^{3}$ A detailed analysis may be found in ref. $[4 \mid$; the tetracritical point arises because we have chosen a section of the fint dimensional space that is tangent to the line of tricriticit points, rather than a section which intersects it separately on odd and even sublattices, and (iii) a one-dimensinal model containing staggered magnetic fields of different wavelength. Both models (ii) and (iii) are exactly soluble $|5|$ In addition to these nod. of systems, several experimental systems may have critical points of order greates than three. For exitmple. the order of the "iriple point" of the spin-thop transition is at least three, but whether it is truly three or fout has yel to be determined.

In conclusion. it should be moted that there are also nom-geometrical ways of defining order which are comnected with the scaling hypothesis. The order of at space of critical points tums out to be equal to the number of relevant scaling variables about each point of that space. In most of the examples known to the authors, the two definitions coincide; in particular. this is true for the examples mentioned above. in which eq. (1) is satisfied as an equality. The definition if order in terms of the number of relevant scaling variables may be more appropriate in very complex systems such as fluid mixtures with more than three components.

\section{References}

11) R.B. Griftiths and J.C. Wheeler. Phys. Rev. A2 (1970) 1047 .

(2) R.B. Griffiths, Phys. Rev, Lett. 24 (1970) 715.

13| I.F. Nagle. Phys. Rev. A2 (1970) 2124: J.F. Nagle and J.C. Bonner. J. Chem. Phys. 54 (1971) 724 ;

J.C. Bonner and J.l. Nagle. J. Appl. Phys. 42 (1971) 1280 .

14) A. Hankey, Ph. D. thesis 1973

15] A. Hankey, T.S. Chang and H.L. Stanley. A.I.P. Conterence Publication ne. 10 (Proc. 1972 Conf. on Magnetism and magnetic materials, Denver. Colorado, USA). 